\title{
Cost-Effectiveness of Global Endometrial Ablation vs. Hysterectomy for Treatment of Abnormal Uterine Bleeding: US Commercial and Medicaid Payer Perspectives
}

\author{
Jeffrey D. Miller, MS, ${ }^{1}$ Gregory M. Lenhart, MS, Machaon M. Bonafede, PhD, MPH, \\ Andrea S. Lukes, MD, MHSc, ${ }^{2}$ and Shannon K. Laughlin-Tommaso, MD, MPH ${ }^{3}$
}

\begin{abstract}
Cost-effectiveness modeling studies of global endometrial ablation (GEA) for treatment of abnormal uterine bleeding (AUB) from a US perspective are lacking. The objective of this study was to model the costeffectiveness of GEA vs. hysterectomy for treatment of AUB in the United States from both commercial and Medicaid payer perspectives. The study team developed a 1-, 3-, and 5-year semi-Markov decision-analytic model to simulate 2 hypothetical patient cohorts of women with AUB-1 treated with GEA and the other with hysterectomy. Clinical and economic data (including treatment patterns, health care resource utilization, direct costs, and productivity costs) came from analyses of commercial and Medicaid claims databases. Analysis results show that cost savings with simultaneous reduction in treatment complications and fewer days lost from work are achieved with GEA versus hysterectomy over almost all time horizons and under both the commercial payer and Medicaid perspectives. Cost-effectiveness metrics also favor GEA over hysterectomy from both the commercial payer and Medicaid payer perspectives-evidence strongly supporting the clinical-economic value about GEA versus hysterectomy. Results will interest clinicians, health care payers, and self-insured employers striving for cost-effective AUB treatments. (Population Health Management 2015;18:373-382)
\end{abstract}

\section{Introduction}

A BNORMAL UTERINE BLEEDING (AUB) encompasses heavy, prolonged, or excessive menstrual bleeding that is bothersome to a woman and interferes with physical, emotional, social, and/or material quality of life. ${ }^{1-9}$ Prevalence of AUB ranges from $10 \%$ to $30 \%$ among women of reproductive age, affecting more than 10 million women in the United States each year. ${ }^{9-12}$ Nearly one third of all gynecologic office visits are related to $\mathrm{AUB},{ }^{2,8,13}$ with estimated annual direct costs of AUB in the United States ranging from $\$ 1$ to $\$ 1.55$ billion and indirect costs from $\$ 12$ to $\$ 36$ billion. $^{12}$

Hysterectomy cures AUB, but can cause significant morbidity, typically requires a long recovery period, has high associated health care costs, and in rare instances can result in death. ${ }^{7,9,14}$ Endometrial ablation is a minimally invasive surgical alternative for women who want to preserve their uterus, and has lower morbidity, cost, and recovery time compared to hysterectomy. ${ }^{14}$ Global endometrial ablation (GEA), a second-generation technique, can be performed without general anesthesia and typically in an outpatient office setting. ${ }^{3,7,8,15}$ However, GEA does have some associated complications and risks of reintervention. The hysterectomy rates for women after GEA range between $2 \%$ and $21 \% .^{3,16-27}$

GEA is particularly appealing in today's cost-conscious health care environment, which values safe and efficacious treatments that reduce the costs associated with inpatient care. ${ }^{15}$ Numerous cost-effectiveness modeling studies of AUB treatment have been conducted over the past decade, but only a few have compared GEA and hysterectomy, and predominately from a UK perspective. ${ }^{28-32}$ From a US perspective, it remains unclear whether GEA is cost-effective when compared

\footnotetext{
${ }^{1}$ Truven Health Analytics, Cambridge, Massachusetts.

${ }^{2}$ Carolina Women's Research and Wellness Center, Durham, North Carolina.

${ }^{3}$ Department of Obstetrics \& Gynecology, Mayo Clinic, Rochester, Minnesota.
}

(C) Miller et al. 2015; Published by Mary Ann Liebert, Inc. This Open Access article is distributed under the terms of the Creative Commons License Attribution-Non-Commercial Share Alike (http://creativecommons.org/licenses/by-nc-sa/4.0/). 
with hysterectomy. The objective of this study was to model the cost-effectiveness of GEA vs. hysterectomy for treatment of AUB in the United States from both commercial and Medicaid payer perspectives.

\section{Methods}

\section{Model structure and target population}

A decision-tree, state-transition (semi-Markov) model was developed in TreeAge Pro 2012 (TreeAge Software, Inc., Williamstown, MA) to simulate 2 hypothetical patient cohorts of women with AUB: one treated with GEA and the other with hysterectomy (Fig. 1). The approach is consistent with other economic models of AUB treatment with GEA and hysterectomy that have been developed in recent years. $^{28-32}$ Two versions of the model were created: one containing clinical and economic data oriented from the US commercial health care payer perspective and the other oriented from a US Medicaid perspective. Although the underlying clinical and cost data are different, the structure and functional operation of the 2 versions of the model are identical, with the exception that the commercial payer perspective model can generate outputs associated with work impairment. Comparisons among these 2 payer types account for the fundamental differences in reimbursement rates $(27 \%$ to $65 \%$ less for Medicaid than commercial health plans, according to a recent Government Accountability
Office report) ${ }^{33}$ but also provide important insights into the inherent differences in the patients themselves, who tend to differ with regard to demographics, economic status, health status, treatment-seeking behaviors, and the types and quality of clinicians and health care institutions providing for their medical needs.

As shown in Figure 1, simulations begin at the point where AUB treatment is initiated. The focus of the modeling analyses was specifically on the stratum of premenopausal women for whom childbearing is complete and who seek a permanent, nonreversible, 1-time treatment option for their AUB with the 2 choices of GEA or hysterectomy. It is implicit that these women may have used medical management (eg, levonorgestrel intrauterine system [LNG-IUS], pharmacological therapy) prior treatment for their AUB, which is consistent with treatment guidelines. ${ }^{1}$ In some cases, these other forms of treatment may have failed or, for a variety of reasons, were not a viable initial option. Regardless, the model analyses initiate on the specific day when each woman in the simulated cohorts underwent her GEA or hysterectomy procedure. Average starting age in both cohorts was assumed to be 42 years, consistent with the underlying clinical and economic data ${ }^{34-36}$ and demographically similar to cohorts of women in other economic models of surgical AUB treatment. ${ }^{28-32}$ The cohorts were modeled over a time horizon of 1 to 5 years in monthly cycles, with the model capable of generating results in any

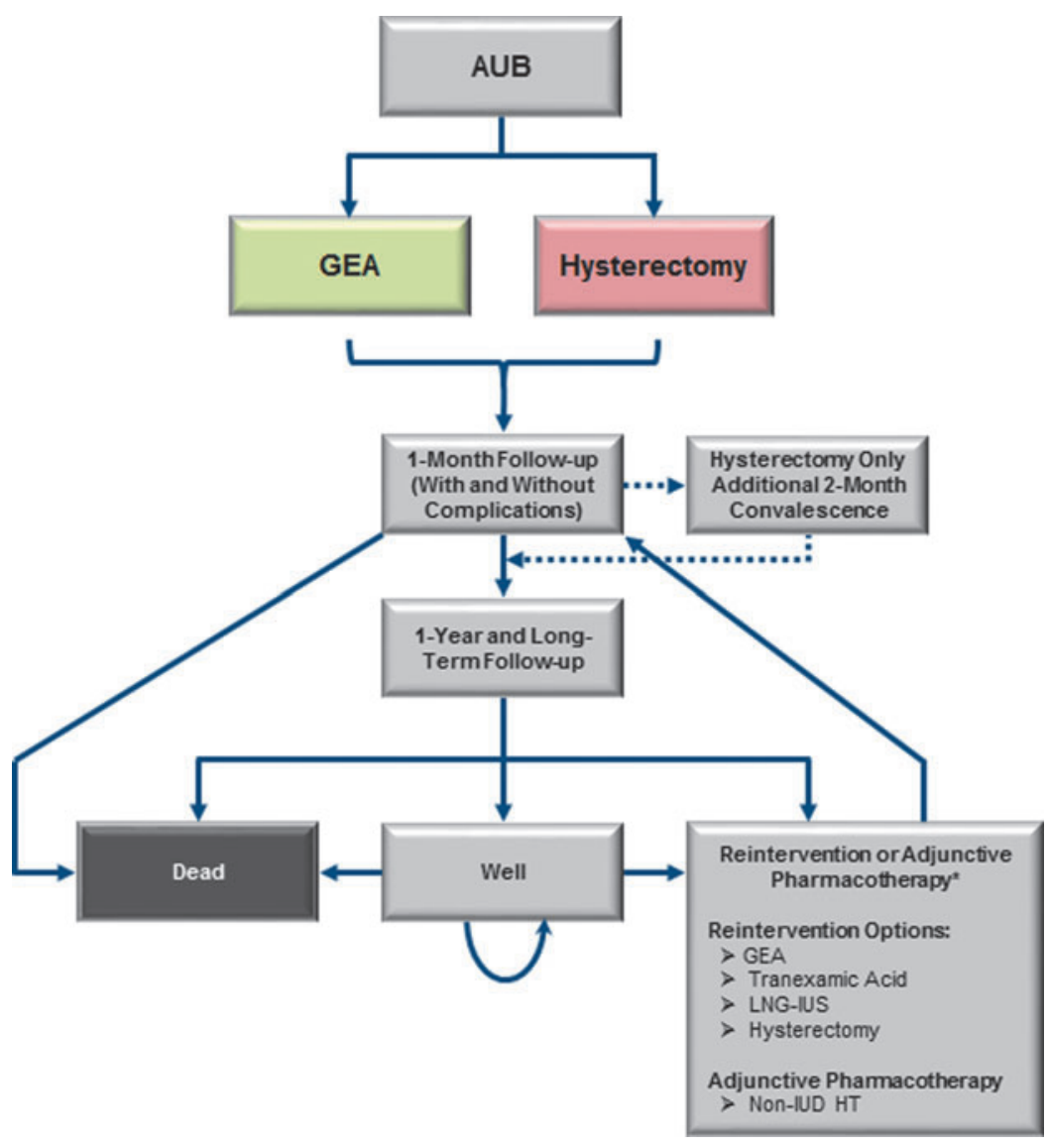

FIG. 1. Clinical pathways within the cost-effectiveness model. AUB, abnormal uterine bleeding; GEA, global endometrial ablation; HT, hormone therapy; IUD, intrauterine device; LNG-IUS, levonorgestrel intrauterine device. 
Table 1. Cost-Effectiveness Model Health States

Intervention

1. Intervention and 1-month follow-up without complication

2. Intervention and 1-month follow-up with complication

3. Post intervention; well

4. Post hysterectomy convalescence ( 2 additional months beyond initial 1-month follow-up for hysterectomy treatment arm only)

Reintervention (GEA treatment arm only)

5. Reintervention with GEA and 1-month follow-up without complication

6. Reintervention with GEA and 1-month follow-up with complication

7. Post reintervention with GEA; well

8. Reintervention with tranexamic acid and 1-month follow-up without complication

9. Reintervention with tranexamic acid and 1-month follow-up with complication

10. Post reintervention with tranexamic acid; well

11. Reintervention with LNG-IUS and 1-month follow-up without complication

12. Reintervention with LNG-IUS and 1-month follow-up with complication

13. Post reintervention with LNG-IUS; well

14. Reintervention with hysterectomy and 1-month follow-up without complication

15. Reintervention with hysterectomy and 1-month follow-up with complication

16. Post reintervention with hysterectomy; well

17. Post reintervention hysterectomy convalescence ( 2 additional months beyond initial 1-month follow-up)

Adjunctive/alternative pharmacotherapy following intervention or reintervention (GEA treatment arm only)

18. Initiate adjunctive pharmacotherapy (ie, Non-IUD HT) and 1-month follow-up without complication

19. Initiate adjunctive pharmacotherapy (ie, Non-IUD HT) and 1-month follow-up with complication

20. Adjunctive pharmacotherapy; well

Death

21. Dead

GEA, global endometrial ablation; HT, hormonal therapy; IUD, intrauterine device; LNG-IUS, levonorgestrel intrauterine system.

given month. Menopause was not factored into the model calculations, also consistent with other economic models of surgical AUB treatment.

Twenty-one health states were included in the model, as listed in Table 1, comprising intervention with GEA or hysterectomy (accounting for occurrence of complications), post-GEA reintervention with secondary GEA, tranexamic acid, or LNG-IUS because of persistent or recurrent AUB or general dissatisfaction with initial intervention (again, accounting for complications), and use of adjunctive pharmacotherapy following GEA (again, accounting for complications), as well as a small probability of death from hysterectomy surgery or actuarial death from all other causes. In any given model cycle, the patient cohorts either remain in or move between discrete health states to replicate the natural course of postsurgical AUB treatment over time. Time-varying transition probabilities, costs, and quality-oflife values are ascribed to each health state and differ according to the characteristics of the particular treatment options being compared. At the end of each simulation, differences in cumulative total costs and outcomes between treatment cohorts generate estimates of cost-effectiveness.

Economic modeling guidelines published by the International Society for Pharmacoeconomics and Outcomes Research $^{37}$ and the Academy of Managed Care Pharmacy ${ }^{38}$ were followed throughout the model design and development process.

\section{Data inputs and sources}

Data inputs for the model and the sources from which they were derived are listed in online Supplemental Tables S1-S6 (available online at www.liebertpub.com/pop).
Clinical and economic modeling data (including treatment patterns, health state transition probabilities, health care resource utilization, direct costs, and productivity costs) were derived primarily from de novo analyses of 3 large medical claims databases: (1) the Truven Health MarketScan Commercial Claims and Encounters Database; (2) the Truven Health MarketScan Medicaid Multi-State Database; and (3) the Truven Health MarketScan Health Productivity and Management Database. ${ }^{34-36}$ These databases provide information on individuals covered by a variety of employersponsored private health insurance plans and employer-paid Medicare supplemental insurance and are considered nationally representative of persons with employer-sponsored health insurance with respect to geography, age, and sex. All patient-level and provider-level data within the MarketScan databases contain synthetic identifiers to protect the privacy of individuals and data contributors, and the data have been verified to be fully compliant with the Health Insurance Portability and Accountability Act of 1996.

The database analyses underlying this study focused in a naturalistic, real-world manner on 63,482 women aged 30-55 years with diagnosed AUB who initiated treatment with GEA or hysterectomy (index event) during 20062010. Twelve months of continuous enrollment pre and post index event were required, and health care utilization and costs were assessed in the year following treatment initiation. Probabilities and costs of reintervention were tracked for 3 years following GEA. Workplace absenteeism and short-term disability costs were reported for a subset of patients from the commercial database analyses. A more detailed description of the methods and results of these database analyses have been published elsewhere. $^{34-36}$ 
The small amount of data pertaining to utilities and mortality not obtainable from these database analyses were derived from published literature and various other published and unpublished sources, as documented in Supplemental Table S4 and Supplemental Table S6.

Interventions and reinterventions. Different types of GEA could not be discerned from coding of GEA payment claims within the MarketScan Research Databases, so GEA was modeled as a composite of all GEA technologies. Firstgeneration endometrial ablation techniques were excluded from the data analyses. Similarly, hysterectomy was modeled as a composite of laparoscopic/robotic-assisted hysterectomy, vaginal hysterectomy, and abdominal hysterectomy as in realworld practice. Based on estimates derived from the MarketScan database analyses, a small probability of reintervention with LNG-IUS was allowed in the model. Similarly, tranexamic acid also was included in the model as a reintervention option, but limited available claims data for tranexamic acid necessitated substituting data pertaining to non-intrauterine device (IUD) hormonal therapies as a proxy. Use of adjunctive/alternative pharmacotherapy with non-IUD hormonal therapy, comprising the large array of available combination oral contraceptives, conjugated estrogens, and progestin-only pills was accounted for in the model calculations as well. As summarized in Supplemental Table S2, 3 sets of reintervention probabilities and reintervention-type distributions are featured in the model pertaining to the 3 sequential years after initial AUB intervention. All values were held constant at the third-year values in the fourth and fifth year of the analyses.

Complications. Probabilities of complications in the first month (30 day) post intervention or reintervention are listed in Supplemental Table S3. Complications included: cervical occlusion, cervical trauma (including cervical lacerations and hematometra), uterine perforation, bowel perforation, fluid overload, pregnancy, pyometra, and device complications; a subsequent AUB treatment within 30 days; an inpatient stay or emergency room visit within 2 days of the intervention or reintervention procedure (excluding patients with an inpatient stay for that procedure). Longer term ( $>30$ days) complications following hysterectomy, such as pelvic organ prolapse, which has a high incidence of 1.3 per 1000 women-years, ${ }^{39}$ were not classified as "complications," although their associated costs were fully accounted for in the model calculations. All complications data were derived from the MarketScan database analyses.

Costs. Direct costs of intervention and 30-day follow-up for GEA and hysterectomy are shown in Supplemental Table S1, as are the reintervention and 30-day direct costs. All cost estimates were derived from the MarketScan database analyses and shown in 2014 US dollars. These costs are actual payments to providers from adjudicated insurance claims, and are not billed charges.

Intervention/reintervention costs and associated 30-day follow-up costs encompass "all health care expenditure," not just gynecological-related health care expenditure. However, chronic monthly costs for patients in a postintervention/ reintervention "well" health state are specifically gynecological-related health care expenditures. All postintervention costs are assumed to remain constant indefinitely within a simulation, changing only when reintervention is encountered and new post-reintervention costs are ascribed.

Health state utility values. Health state utility scores (between $0=$ death, and $1=$ perfect health) were used to calculate quality-adjusted life-years (QALYs). As documented in Supplemental Table S4, utility data were derived from other published economic models of AUB that had obtained estimates from patient-reported outcomes studies with quality-of-life assessments. Most of the utility values used in the analyses originate from the studies by Sculpher et $\mathrm{al}^{40}$ and Hurskainen et al. ${ }^{41}$ Sculpher et al derived utility values from interviews with 60 women with menorrhagia using the well-regarded time trade-off (TTO) technique. Women in the study were presented with a set of scenarios describing health states relating to menorrhagia and treatment satisfaction and were asked to make TTO choices between being in perfect health for a set amount of time compared with being in less than perfect health for a variable amount of time. Hurskainen et al conducted a large randomized clinical trial of 236 women with menorrhagia and estimated utility values for various states relating to menorrhagia and treatment satisfaction using the EQ-5D, a standardized instrument for measuring health-related quality of life.

Work impairment. Work impairment was defined in the model as all-cause absence from work and work loss related to short-term disability. Supplemental Table S5 shows the monthly probability of work absence or work loss from short-term disability and the corresponding number of days of lost work. Absenteeism costs were calculated in the analyses as the number of hours of reported absence multiplied by a constant average wage of $\$ 30$ per hour. ${ }^{42-44}$ A wage constant of $\$ 21$ per hour was applied to short-term disability claims to reflect the general practice of employers covering $70 \%$ of normal wages during a period of disability. Data were only available to evaluate work impairment under the commercial health care payer perspective, not the Medicaid perspective.

\section{Model outputs}

Model analysis outcomes. Analytical results generated by the model include an assortment of comparative clinical and economic outcomes, with presentation of incremental cost-effectiveness ratios (ICERs) for GEA vs. hysterectomy. Clinical outcomes include total and incremental QALYs, and total and incremental cases of treatment complications. Work productivity outcomes (US commercial payer perspective only) included total and incremental number of work days lost related to worker absence and short-term disability. Economic outcomes include total and incremental direct costs related to worker absence and short-term disability. Cost-effectiveness outcomes include cost per QALY, cost per complication case avoided, and cost per work day saved.

Analysis time horizon. Although the model was programmed to simulate any time horizon from 1 month up to 5 years (and 10 years as a sensitivity analysis), a few key time points were selected that would be of universal interest: 1 year, 3 years, and 5 years. 
Sensitivity analyses. A variety of 1-way and probabilistic sensitivity analyses (PSA) were conducted to test the robustness of model parameter values and their impact on the ICERs. Using a second-order Monte-Carlo simulation (1000 iterations), PSA was conducted on the model's primary cost-effectiveness outcome, incremental cost per incremental QALY. The parameter values of each probability distribution were calculated from the mean and standard error of the model input parameters. By drawing randomly from those distributions, a large number of estimates of costs and QALYs for GEA versus hysterectomy were generated, thus testing the consequences of varying input parameters (45). Targeted 1-way deterministic sensitivity analyses were performed on the ICERs featuring complications and work productivity. A 10-year analysis scenario was performed to test robustness of the model estimates over long time horizons.

Discounting. All cost inputs and outputs from the model are in 2014 US dollars. In the base case scenario, both costs and survival estimates (ie, QALYs) were discounted annually at a discount rate of $3 \%$, as supported by pharmacoeconomic guidelines published by the Academy of Managed Care Pharmacy. ${ }^{38}$

\section{Results}

Analysis results for 1-, 3-, and 5-year scenarios from both the commercial payer and Medicaid payer perspectives are summarized in Table 2. One-year direct costs of GEA were $\$ 7352$ and $\$ 6306$ in the commercial payer and Medicaid payer perspectives, respectively, which was about half of the corresponding costs of hysterectomy. The cost differential between the treatments closed somewhat over time, but even at 5 years GEA costs were still one third less than hysterectomy costs (Fig. 2).

Clinical outcomes (ie, occurrence of intervention/reintervention complications) and work impairment outcomes are also shown in Table 2; the differences between GEA and hysterectomy varied over time. Greater disutility (ie, lost quality of life) associated with hysterectomy versus GEA (which led to lower QALYs in the short term) shifted to favor hysterectomy by about 1.5 years in both the commercial payer and Medicaid payer perspective analyses, mostly because of the inherently higher long-term utility weights assigned to hysterectomy, but also from the cumulative disutility experienced by GEA patients undergoing repeat ablation and other forms of reintervention avoided by hysterectomy patients. The difference in QALYs between GEA and hysterectomy remains small-even by year 5, QALYs are only about 3\% (0.12 QALYs) lower for GEA. Intervention/reintervention complication rates were substantially lower for GEA versus hysterectomy: Whereas $35.6 \%$ of hysterectomy patients had complications in the first year under the commercial payer perspective, only $17.1 \%$ of GEA patients had complications. The gap narrowed over time as the initial complications of hysterectomy remained constant while reintervention complications for GEA patients gradually accumulated. However, even by year 5 GEA patients had $41 \%$ fewer complications than hysterectomy patients. Shortterm results were similar under the Medicaid perspective, but by 5 years intervention/reintervention complications of GEA exceeded those for hysterectomy by about $1.6 \%$.
Over the 5-year time horizon, direct costs of GEA were lower than those of hysterectomy in both the commercial payer and Medicaid perspectives (Table 2). In the commercial payer analysis, the indirect costs of GEA also were lower than for hysterectomy, with 38.5 work days lost for GEA versus 55.3 days for hysterectomy, corresponding with indirect costs of $\$ 8976$ versus $\$ 13,087$. Additionally, GEA was associated with fewer complications than hysterectomy in the commercial payer perspective: $21.1 \%$ of GEA patients had composite intervention/reintervention complications versus $35.6 \%$ of hysterectomy patients. Much higher rates of composite intervention/reintervention complications were found in the Medicaid payer perspective, and complications for GEA patients were slightly higher than for hysterectomy patients (Table 2). Although GEA cost less than hysterectomy and resulted in fewer complications in the commercial perspective, it also garnered fewer QALYs (Table 2).

Cost-effectiveness ratios generated by the model appear in Table 2. In the 1-year scenario for both the commercial payer and Medicaid perspectives, GEA "dominated" hysterectomy, meaning that it both cost less and conferred more QALYs. Although favorable cost savings for GEA were upheld through the 5-year scenario, GEA conferred fewer QALYs in the 3and 5-year scenarios, resulting in decremental cost-effectiveness ratios, meaning that GEA promotes cost savings but is less effective in terms of conferring QALYs. Decremental costeffectiveness ratios are asymmetric with the more conventional ICERs, which are intended to calculate an amount of money spent for every QALY gained. Under the commercial payer perspective, GEA saved \$99,311 per QALY lost in the 3year scenario and saved $\$ 41,186$ per QALY lost in the 5-year scenario. Under the Medicaid payer perspective, GEA saved $\$ 106,627$ per QALY lost in the 3-year scenario and $\$ 42,898$ per QALY lost in the 5-year scenario.

\section{Sensitivity analyses}

Overall, varying inputs to the model did not appreciably alter the ICERs (including economic dominance), nor did they affect conclusions about the cost-effectiveness of GEA relative to hysterectomy. Sample results are shown on the cost-effectiveness planes in Supplemental Figure S1 and Supplemental Figure S2, where the 5-year scenario ICERs are plotted for the commercial payer and Medicaid payer perspectives, respectively. GEA always costs less than hysterectomy, even under the extremes of probabilistic sampling. Most (68\%) of the ICERs plotted into the lower left quadrant of the plane where both costs and QALYs are lower than hysterectomy. However, nearly one third (32\%) of the probabilistically generated ICERs are in the lower right quadrant of the plane where GEA dominates hysterectomy by simultaneously conferring cost savings and more QALYs.

A special 10-year scenario simulation was performed to explore results projected over a long time horizon. As seen in the 5-year scenario, incremental cost differences remained highly favorable for GEA in both the commercial payer and Medicaid perspectives. Indirect costs attributable to work absence and short-term disability leave in the commercial payer perspective also remained advantageous. Hysterectomy conferred a slightly greater number of QALYs at 10 years, thus saving about $\$ 14,000$ per QALY lost in both payer perspectives. Economic dominance of GEA versus hysterectomy in 


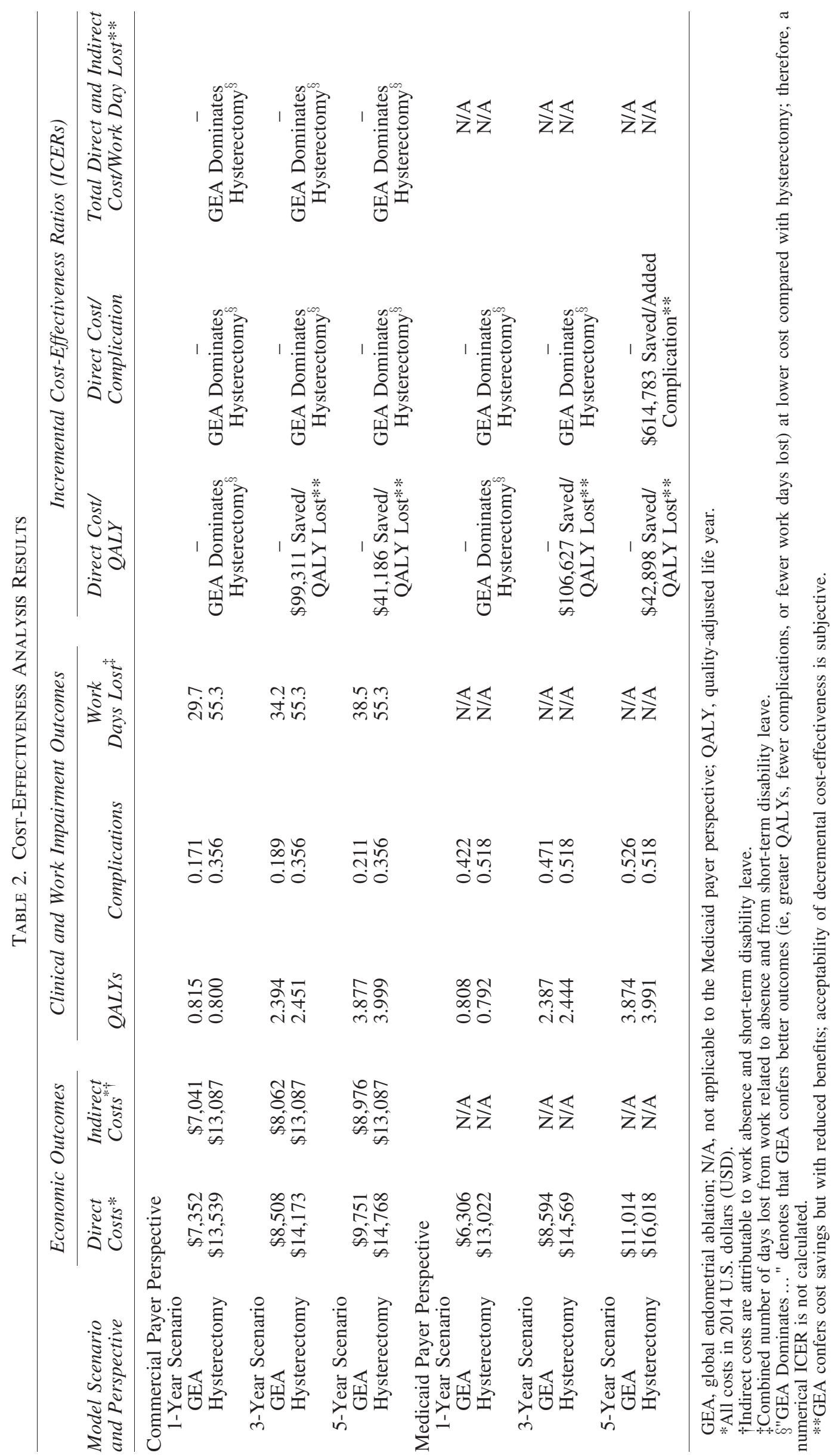




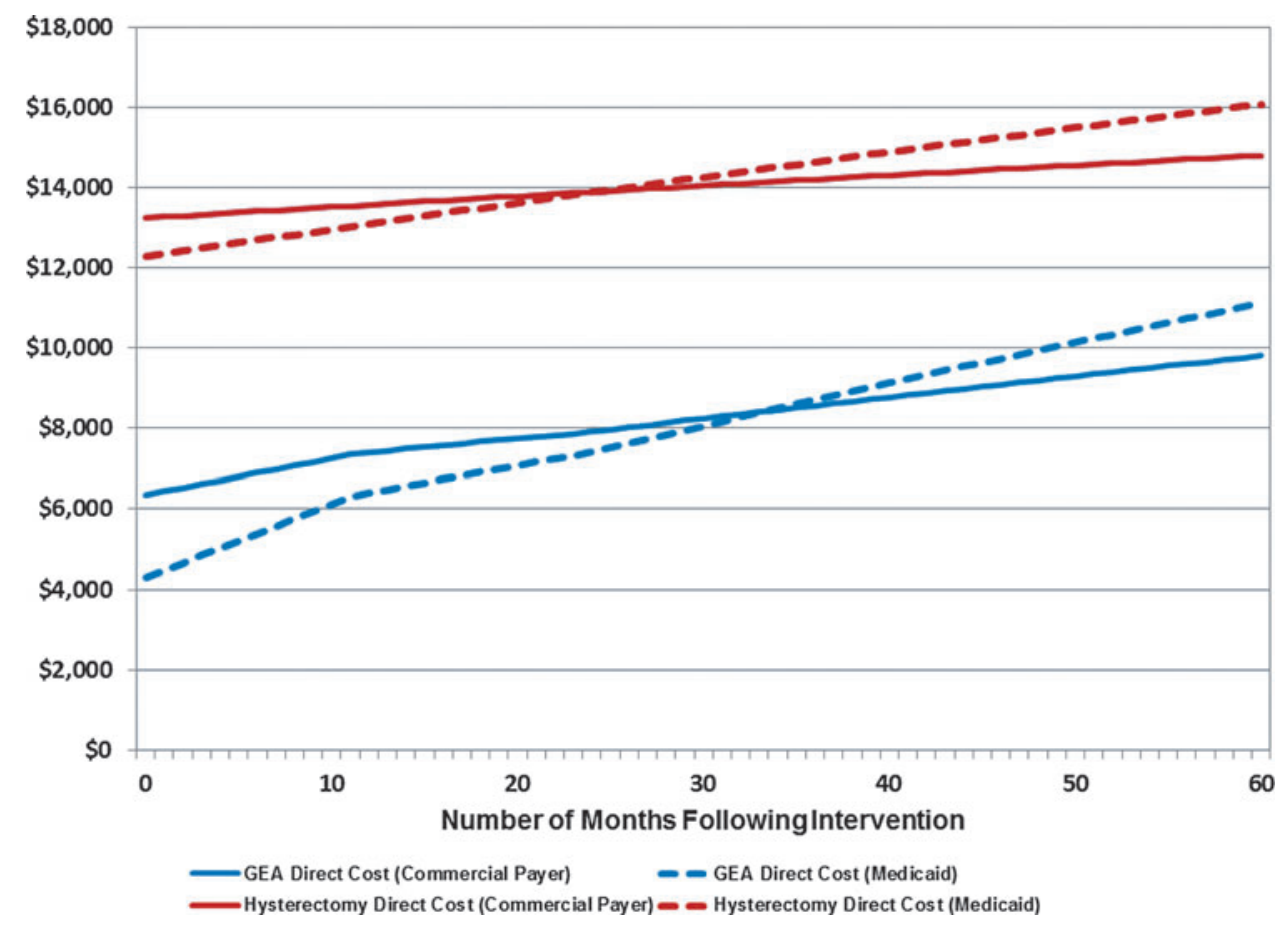

FIG. 2. Direct cost over time by treatment and payer perspective. GEA, global endometrial ablation.

terms of cost-per-complication avoided was maintained at 10 years in the commercial payer perspective. In the Medicaid perspective, at 10 years, GEA saved about $\$ 28,000$ per added complication. Unchanging from the 5-year scenario, GEA continued to dominate hysterectomy in the 10 -year commercial payer analysis in terms of costs and lost work days.

\section{Discussion}

\section{Principal findings}

This model estimates that GEA has substantial cost superiority over hysterectomy over short (1-year) and longer (5year) time horizons, and under both commercial payer and Medicaid perspectives. Although short-term cost savings are highest, by the fifth year post intervention, direct costs for GEA patients are still about one third less $(\approx \$ 5000)$ than for hysterectomy patients. The same is true for indirect costs, under the commercial payer perspective. Combining these cost savings with comparative treatment benefits yielded costeffectiveness metrics favoring GEA over hysterectomy as an AUB treatment strategy. In the first 18 months immediately following surgical intervention, GEA was found to economically dominate hysterectomy-that is, GEA both costs less and yields greater QALYs and fewer complications. However, over some of the longer time horizons modeled, it was found that cost savings of GEA are achieved in exchange for fewer QALYs and more complications (eg, the 5-year Medicaid scenario), principally from incidence of post-GEA reinterventions. Certainly, patient quality of life is an important consideration in evaluating AUB treatment strategies, although US health care payers tend to view QALYs as a descriptive rather than prescriptive tool, with relegation as only one of multiple inputs for consideration in coverage and payment decisions. In reality, few US payers include a cost-
per-QALY threshold in their decision-making process, relying instead on more actionable metrics such as absolute and incremental costs and net budget impact to inform coverage decision making. ${ }^{45-48}$ Results of the current model analyses demonstrate strong favorability for GEA in this regard.

\section{Comparison with UK cost-effectiveness studies}

Although US and UK perspectives on AUB treatment and associated costs are decidedly different, some comparisons of the current analyses results with those of previously published UK cost-effectiveness modeling studies are interesting to note. For the most part, the UK studies focus on cost-perQALY ICERs, which was only one type of cost-effectiveness metric generated by the current model. Nonetheless, the present study showed GEA dominating hysterectomy in the 1-year scenarios (ie, GEA both cost less and conferred more QALYs), and decremental cost-effectiveness ratios in the 3and 5-year analyses ranging from $\$ 41,000$ to $\$ 107,000$ saved per QALY lost. In the 5-year scenarios presented by Clegg et $\mathrm{al},{ }^{30}$ microwave and thermal balloon endometrial ablation cost less than hysterectomy and conferred more QALYs and, therefore, dominated hysterectomy. Bhattacharya et $\mathrm{al}^{28}$ found second-generation endometrial ablation to be dominated by hysterectomy in one 10-year analysis scenario, but showed decremental cost-effectiveness ( $£ 970$ saved per QALY lost) in an alternative scenario, which was duplicated by Roberts et al. ${ }^{29}$ Garside et al ${ }^{31-32}$ also showed decremental cost-effectiveness for microwave and thermal balloon endometrial ablation versus hysterectomy in a 5-year analysis ( $£ 1006$ saved per QALY lost to $£ 4656$ saved per QALY lost) and in a 10-year analysis ( $£ 2108$ saved per QALY lost to $£ 2410$ saved per QALY lost).

Interpretation of decremental cost-effectiveness metrics such as these are highly subjective and the study team does 
not attempt to impose conclusions about their acceptability from a US health care perspective. However, as health care payers struggle to control medical expenditures, treatment alternatives are important and can become attractive, even if they come with reduced benefits. ${ }^{50-51}$ Technologies capitalizing on favorable cost-quality trade-offs are ubiquitous in other markets (eg, automobiles, computers, furniture). ${ }^{50}$

\section{Work impairment (indirect costs)}

From an employer's perspective, AUB is an expensive condition, and choice of treatments can profoundly affect the direction of those costs. ${ }^{34,52}$ Results generated by the current model indicate that in the first year following surgical treatment of AUB, GEA accounts for almost 26 fewer days of work absence and short-term disability leave compared with hysterectomy, leading to an indirect cost savings of greater than $\$ 6000$. The incremental difference narrows over time, but even by 5 years GEA accounts for more than $30 \%$ fewer days of work loss and associated indirect costs. With a conservative estimate of the annual indirect economic costs of AUB in the US at $\$ 12$ billion, ${ }^{12}$ impaired work productivity related to AUB has substantial economic implications, both for individual women and for society. Very few studies have assessed the impact of AUB treatments on work productivity; most evaluations have focused on work impact of AUB itself. ${ }^{12,52-55}$ although a few recent studies have evaluated the work impact of AUB pharmacotherapies. ${ }^{56-57}$ Estimates generated by the current model do not account for reduced on-the-job productivity.

\section{Implications for Medicaid expansion and Affordable Insurance Exchanges}

Changes to the US Medicaid program have occurred in the context of the federal mandates from the Patient Protection and Affordable Care Act (PPACA) for Medicaid expansion and the creation of new state-based and federally facilitated competitive marketplaces, or Affordable Insurance Exchanges. About $12 \%$ of US women aged 18-64 years rely on Medicaid for their health care coverage, and Medicaid expansion under PPACA is expected to expand eligibility to an additional 7 million currently uninsured women. ${ }^{58-60}$ Using the average of published AUB prevalence statistics of about $20 \%,{ }^{9-12}$ about 1.4 million newly eligible women in the expanded Medicaid program will have AUB, many of whom will go on to receive costly pharmacologic and surgical treatments. Hysterectomies will be of particular financial concern, not only because of their high cost but also because they comprise about $27 \%$ of the total pharmacologic and surgical AUB interventions that are performed each year in the Medicaid population. ${ }^{35-36}$ Results of the current analyses suggest that GEA may save substantial costs, as much as $\$ 6700$ per patient in the first year following intervention and up to $\$ 5000$ at 5 years. Consequently, state-based and federally facilitated Exchanges may focus intently on GEA (particularly in-office procedures) as a way to reduce costs.

\section{Limitations}

Although the literature provided health-state utility values sufficient for use in the current model, they are mostly from older studies, some of which predate the advent of second- generation endometrial ablation technologies. Notably, the extensively referenced 1998 cost-utility analysis by Sculpher et $\mathrm{al}^{40}$ comparing transcervical resection of the endometrium and abdominal hysterectomy appears to be a common point of origin for most AUB treatment utility values employed in most other models. This has occurred despite the derivation of the data using a TTO technique in a sample of 60 women with complication-free AUB. The current model results were fairly sensitive to changes in utility values, but it is difficult to say how the results would have changed if more current, robust utility data were available, particularly if those data were specific to GEA and the techniques of hysterectomy now commonly employed in the US health care system. Roberts et $\mathrm{al}^{29}$ shared similar misgivings about the lack of current appropriate utility data for contemporary AUB treatments. The current model should be revisited when newer utility values become available.

There were other data limitations in the current study, most of which are inherently tied to using an administrative health care database as a primary data source. First, as observed in other retrospective claims database analyses, the most completely recorded data are those that affect reimbursement. Hence, the capture of expenditures is highly accurate, but data on comorbidities and disease severity may not be as carefully recorded. Second, procedure coding in claims data is limited in its ability to differentiate specific types/techniques of GEA or hysterectomy. Analyses of these different procedure types may have revealed differences in clinical and cost outcomes from the model, but it is not possible to speculate about the magnitude and direction of those differences. Third, because of the structure of the underlying claims database analyses, the study team did not conduct age-stratified subgroup analyses with the model, although patient age may correlate with clinical and economic outcomes. Fourth, the indirect costs included in this analysis are not exhaustive. For example, absenteeism and short-term disability claims do not include caregiver time, child care time, long-term disability, or reduced productivity while at work. Finally, the study population consists of patients with commercial insurance or Medicaid supplemental insurance; therefore, the results may not represent all patients with AUB, especially those uninsured or covered under other Medicaid programs.

\section{Conclusion}

The role of GEA in the surgical treatment of AUB has grown. Despite purported clinical and financial advantages over hysterectomy, little has been reported about the costs and cost-effectiveness of GEA from a US perspective. The current economic model estimates strong financial favorability for GEA versus hysterectomy from both the commercial payer and Medicaid payer perspectives. Over a variety of time horizons, GEA may save costs while reducing treatment complications and lost workdays. From the patient perspective, model results suggest better quality of life in the short term after GEA, while longer term impacts remain unclear. Results from this study will interest US commercial health payers and self-insured employers seeking cost-effective treatment for AUB. Similarly, new federal mandates for Medicaid expansion likely will focus on outpatient treatments like GEA to reduce Medicaid costs. 


\section{Author Disclosure Statement}

Drs. Bonafede, Lukes, and Laughlin-Tommaso, and Mr. Miller and Mr. Lenhart declared no conflicts of interest with respect to the research, authorship, and/or publication of this article.

Funding for this study was provided by Hologic, Inc., Marlborough, MA.

\section{References}

1. American College of Obstetricians and Gynecologists (ACOG), Committee on Practice Bulletins-Gynecology. Practice bulletin no. 136: management of abnormal uterine bleeding associated with ovulatory dysfunction. Obstet Gynecol. 2013 Jul;122:176-185.

2. American Congress of Obstetricians and Gynecologists (ACOG), Committee on Practice Bulletins-Gynecology. Practice bulletin no. 128: diagnosis of abnormal uterine bleeding in reproductive-aged women. Obstet Gynecol. 2012 Jul;120:197-206.

3. Fox KE. Management of heavy menstrual bleeding in general practice. Curr Med Res Opin. 2012;28:1517-1525.

4. Munro MG, Critchley HO, Fraser IS. The FIGO classification of causes of abnormal uterine bleeding: Malcolm G. Munro, Hilary O.D. Crithcley, Ian S. Fraser, for the FIGO Working Group on Menstrual Disorders. Int J Gynaecol Obstet. 2011;113(1):3-13.

5. National Institute for Health and Care Excellence (NICE). Heavy menstrual bleeding: Clinical Guideline 44. January 2007. http://www.nice.org.uk/nicemedia/pdf/CG44FullGuide line.pdf Accessed November 24, 2014.

6. Fraser IS, Critchley HO, Munro MG, Broder M. Can we achieve international agreement on terminologies and definitions used to describe abnormalities of menstrual bleeding? Hum Reprod. 2007;22:635-643.

7. Apgar BS, Kaufman AH, George-Nwogu U, Kittendorf A. Treatment of menorrhagia. Am Fam Physician. 2007;75: 1813-1819.

8. Osei J, Critchley H. Menorrhagia, mechanisms and targeted therapies. Curr Opin Obstet Gynecol. 2005;17:411-418.

9. Oehler MK, Rees MC. Menorrhagia: an update. Acta Obstet Gynecol Scand. 2003;82:405-422.

10. Sweet MG, Schmidt-Dalton TA, Weiss PM, Madsen KP. Evaluation and management of abnormal uterine bleeding in premenopausal women. Am Fam Physician. 2012;85:35-43.

11. Centers for Disease Control and Prevention (CDC). Blood disorders in women: heavy menstrual bleeding. 2011. http:// www.cdc.gov/ncbddd/blooddisorders/women/menorrhagia .html. Accessed November 24, 2014.

12. Liu Z, Doan QV, Blumenthal P, Dubois RW. A systematic review evaluating health-related quality of life, work impairment, and health-care costs and utilization in abnormal uterine bleeding. Value Health. 2007;10(3):183-194.

13. Spencer CP, Whitehead MI. Endometrial assessment revisited. Br J Obstet Gynaecol. 1999;106:623-632.

14. Stovall DW. Alternatives to hysterectomy: focus on global endometrial ablation, uterine fibroid embolization, and magnetic resonance-guided focused ultrasound. Menopause. 2011;18:437-444.

15. Canali C. Global endometrial ablation for menorrhagia: the office option. http://www.obgmanagement.com/uploads/ media/SupplOBG_Omnia1.pdf. Accessed November 24, 2014.

16. Lukes A, Evantash E. The issue of scarring post-ablation: the data. Contemp OB/GYN. 2012;57(11):33-36.
17. Campbell P, Monaghan C, Parker M. NovaSure endometrial ablation: a review of 400 cases. Gynecol Surg. 2012;(9):73-76.

18. Clark TJ, Samuel N, Malick S, Middleton LJ, Daniels J, Gupta JK. Bipolar radiofrequency compared with thermal balloon endometrial ablation in the office: a randomized controlled trial. Obstet Gynecol. 2011;117:109-118.

19. Cooper K, Lee A, Chien P, Raja E, Timmaraju V, Bhattacharya S. Outcomes following hysterectomy or endometrial ablation for heavy menstrual bleeding: retrospective analysis of hospital episode statistics in Scotland. BJOG. 2011;118:1171-1179.

20. Penninx JP, Mol BW, Engels R, et al. Bipolar radiofrequency endometrial ablation compared with hydrothermablation for dysfunctional uterine bleeding: a randomized controlled trial. Obstet Gynecol. 2010;116:819-826.

21. El-Nashar SA, Hopkins MR, Creedon DJ, et al. Prediction of treatment outcomes after global endometrial ablation. Obstet Gynecol. 2009;113:97-106. Erratum in: Obstet Gynecol. 2010;115:663.

22. Ahonkallio S, Martikainen H, Santala M. Endometrial thermal balloon ablation has a beneficial long-term effect on menorrhagia. Acta Obstet Gynecol Scand. 2008;87:107-110.

23. Kleijn JH, Engels R, Bourdrez P, Mol BW, Bongers MY. Five-year follow up of a randomised controlled trial comparing NovaSure and ThermaChoice endometrial ablation. BJOG. 2008;115:193-198.

24. Longinotti MK, Jacobson GF, Hung YY, Learman LA. Probability of hysterectomy after endometrial ablation. Obstet Gynecol. 2008;112:1214-1220.

25. Gallinat A. An impedance-controlled system for endometrial ablation: five-year follow-up of 107 patients. J Reprod Med. 2007;52:467-472.

26. Bongers MY, Bourdrez P, Heintz AP, Brölmann HA, Mol BW. Bipolar radio frequency endometrial ablation compared with balloon endometrial ablation in dysfunctional uterine bleeding: impact on patients' health-related quality of life. Fertil Steril. 2005;83:724-734.

27. Bongers MY, Bourdrez P, Mol BW, Heintz AP, Brölmann HA. Randomised controlled trial of bipolar radio-frequency endometrial ablation and balloon endometrial ablation. BJOG. 2004;111:1095-1102.

28. Bhattacharya S, Middleton LJ, Tsourapas A, et al. Hysterectomy, endometrial ablation and Mirena ${ }^{\circledR}$ for heavy menstrual bleeding: a systematic review of clinical effectiveness and cost-effectiveness analysis. Health Technol Assess. 2011;15(19):iii-xvi, 1-252.

29. Roberts TE, Tsourapas A, Middleton LJ, et al. Hysterectomy, endometrial ablation, and levonorgestrel releasing intrauterine system (Mirena) for treatment of heavy menstrual bleeding: cost effectiveness analysis. BMJ. 2011;342:d2202.

30. Clegg JP, Guest JF, Hurskainen R. Cost-utility of levonorgestrel intrauterine system compared with hysterectomy and second generation endometrial ablative techniques in managing patients with menorrhagia in the UK. Curr Med Res Opin. 2007;23:1637-1648.

31. Garside R, Stein K, Wyatt K, Round A, Price A. The effectiveness and cost-effectiveness of microwave and thermal balloon endometrial ablation for heavy menstrual bleeding: a systematic review and economic modelling. Health Technol Assess. 2004;8(3):iii, 1-155.

32. Garside R, Stein K, Wyatt K, Round A, Pitt M. A costutility analysis of microwave and thermal balloon endometrial ablation techniques for the treatment of heavy menstrual bleeding. BJOG. 2004;111:1103-1114. 
33. United States Government Accountability Office (GAO). Medicaid Payment: Comparisons of Selected Services Under Fee-for-Service, Managed Care, and Private Insurance. GAO report no. GAO-14-533. July 2014. http:// www.gao.gov/products/GAO-14-533 Accessed November 24, 2014.

34. Bonafede MM, Miller JD, Meyer NM, Lenhart GM. Comparative direct and indirect costs of menorrhagia treatment with global endometrial ablation or hysterectomy. Value Health. 2013;16(3):A73.

35. Bonafede MM, Miller JD, Meyer NM, Lenhart GM. Retrospective database analysis of clinical outcomes and costs for treatment of menorrhagia among women enrolled in US Medicaid programs. Value Health. 2013;16(3):A72.

36. Bonafede MM, Miller JD, Laughlin-Tommaso SK, Lukes AS, Meyer NM, Lenhart GM. Retrospective database analysis of clinical outcomes and costs for treatment of abnormal uterine bleeding among women enrolled in US Medicaid programs. Clinicoecon Outcomes Res. 2014;6:423-429.

37. International Society for Pharmacoeconomics and Outcomes Research (ISPOR). Modeling Good Research Practices. http://www.ispor.org/workpaper/Modeling-Good-ResearchPractices-Overview.asp. Accessed November 24, 2014.

38. Academy of Managed Care Pharmacy (AMCP). The AMCP Format for Formulary Submissions Version 3.1: A Format for Submission of Clinical and Economic Evidence of Pharmaceuticals in Support of Formulary Consideration. December 2012. http://amcp.org/WorkArea/DownloadAsset.aspx?id= 16209. Accessed November 24, 2014.

39. Dällenbach P, Kaelin-Gambirasio I, Dubuisson JB, Boulvain M. Risk factors for pelvic organ prolapse repair after hysterectomy. Obstet Gynecol. 2007;110:625-632.

40. Sculpher M. A cost-utility analysis of abdominal hysterectomy versus transcervical endometrial resection for the surgical treatment of menorrhagia. Int $\mathrm{J}$ Technol Assess Health Care. 1998;14:302-319.

41. Hurskainen R, Teperi J, Rissanen P, et al. Clinical outcomes and costs with the levonorgestrel-releasing intrauterine system or hysterectomy for treatment of menorrhagia: randomized trial 5-year follow-up. JAMA. 2004;291:1456-1463.

42. Hawkins K, Wang S, Rupnow MF. Indirect cost burden of migraine in the United States. J Occup Environ Med. 2007;49:368-374.

43. Goetzel RZ, Hawkins K, Ozminkowski RJ, Wang S. The health and productivity cost burden of the "top 10" physical and mental health conditions affecting six large U.S. employers in 1999. J Occup Environ Med. 2003;45:5-14.

44. Lee DW, Ozminkowski RJ, Carls GS, Wang S, Gibson TB, Stewart EA. The direct and indirect cost burden of clinically significant and symptomatic uterine fibroids. J Occup Environ Med. 2007;49:493-506.

45. Briggs AH. Handling uncertainty in cost-effectiveness models. Pharmacoeconomics. 2000;17:479-500.

46. Fanshawe J, Neumann PJ, Bridges JFP, Meletiche DM, White J. Is health economics an un-American activity? http:// www.medaxial.com/articles/Health_Econ_Unamerican.pdf. Accessed November 24, 2014.

47. Holtorf AP, Brixner D, Bellows B, Keskinaslan A, Dye J, Oderda G. Current and future use of HEOR data in healthcare decision-making in the United States and in emerging markets. Am Health Drug Benefits. 2012;5:428-438.
48. Garber AM. Cost-effectiveness and evidence evaluation as criteria for coverage policy. Health Aff (Millwood). 2004;Suppl Web Exclusives:W4-284-296.

49. Bryan S, Sofaer S, Siegelberg T, Gold M. Has the time come for cost-effectiveness analysis in US health care? Health Econ Policy Law. 2009;4 pt 4:425-443.

50. Nelson AL, Cohen JT, Greenberg D, Kent DM. Much cheaper, almost as good: decrementally cost-effective medical innovation. Ann Intern Med. 2009;151:662-667.

51. Kent DM, Fendrick AM, Langa KM. New and dis-improved: on the evaluation and use of less effective, less expensive medical interventions. Med Decis Making. 2004;24:281-286.

52. Jensen JT, Lefebvre P, Laliberté F, et al. Cost burden and treatment patterns associated with management of heavy menstrual bleeding. J Womens Health (Larchmt). 2012;21: 539-547.

53. Pron G, Cohen M, Soucie J, et al. The Ontario Uterine Fibroid Embolization Trial. Part 1. Baseline patient characteristics, fibroid burden, and impact on life. Fertil Steril. 2003;79:112-119.

54. Côté I, Jacobs P, Cumming D. Work loss associated with increased menstrual loss in the United States. Obstet Gynecol. 2002;100:683-687.

55. Hurskainen R, Aalto AM, Teperi J, et al. Psychosocial and other characteristics of women complaining of menorrhagia, with and without actual increased menstrual blood loss. BJOG. 2001;108:281-285.

56. Wasiak R, Filonenko A, Vanness DJ, et al. Impact of estradiol valerate/dienogest on work productivity and activities of daily living in women with heavy menstrual bleeding. J Womens Health (Larchmt). 2013;22:378-384.

57. Wasiak R, Filonenko A, Vanness DJ, et al. Impact of estradiol-valerate/dienogest on work productivity and activities of daily living in European and Australian women with heavy menstrual bleeding. Int J Womens Health. 2012;4: 271-278.

58. American Congress of Obstetricians and Gynecologists (ACOG). Protect Medicaid and Women's Health. 2012. http://www.acog.org/ /media/Departments/Government\% 20Relations\%20and\%20Outreach/2012ProtectMedicaid.pdf. Accessed November 24, 2014.

59. National Women's Law Center. What the Medicaid Eligibility Expansion Means for Women. 2012. http://www.nwlc .org/sites/default/files/pdfs/what_the_medicaid_eligibility_ expansion_means_for_women_10-23-12.pdf. Accessed November 24, 2014.

60. Kenney GM, Zuckerman S, Dubay L, et al. Opting in to the Medicaid expansion under the ACA: who are the uninsured adults who could gain health insurance coverage? August 2012. http://www.urban.org/UploadedPDF/412630-optingin-medicaid.pdf. Accessed November 24, 2014.

Address correspondence to: Jeffrey D. Miller Truven Health Analytics 150 CambridgePark Drive, $2^{\text {nd }}$ Floor Cambridge, MA 02140

E-mail: jeffrey.d.miller@truvenhealth.com 